Arq. Bras. Med. Vet. Zootec., v.67, n.1, p.140-148, 2015

\title{
Desempenho produtivo e econômico de três grupos genéticos de bovinos recriados a pasto com suplementação e terminados em confinamento
}

\author{
[Productive and economical performance of three genetic groups of animals raised on pasture \\ with supplementation and feedlot] \\ F.S. Moreira ${ }^{1}$, M.M.N.F. Oliveira ${ }^{2}$, S.D.J. Villela ${ }^{2}$, F.A. Barbosa ${ }^{3}$, \\ M.H.F. Mourthé $e^{4}$ F.B. Diniz
}

\footnotetext{
${ }^{1}$ Aluno de pós-graduação - Universidade Federal dos Vales do Jequitinhonha e Mucuri - UFVJM - Diamantina, MG ${ }^{2}$ Universidade Federal dos Vales do Jequitinhonha e Mucuri - UFVJM - Diamantina, MG

${ }^{3}$ Escola de Veterinária - Universidade Federal de Minas Gerais - UFMG - Belo Horizonte, MG

${ }^{4}$ Instituto de Ciências Agrárias - Universidade Federal de Minas Gerais - UFMG - Montes Claros, MG
}

\section{RESUMO}

Avaliou-se o desempenho produtivo e econômico de três grupos genéticos de bovinos em sistema com recria a pasto e terminação em confinamento, com ciclo de produção de um ano. Foram utilizados 36

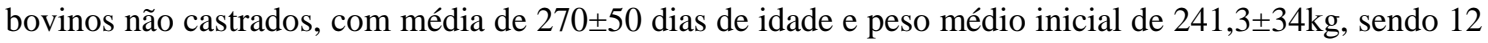
Guzolandos (1/2 Guzerá x 1/2 Holandês), 12 Guzonel (1/2 Guzerá x 1/2 Nelore) e 12 Tricross (1/2 Simental x 1/2 Guzonel). O delineamento estatístico foi inteiramente ao acaso. Os animais permaneceram em pastejo rotacionado de Brachiaria brizantha cv. Marandu, com suplementação comercial; na terminação, receberam dieta composta por silagem de sorgo e concentrado (50:50) durante 61 dias. Os índices de desempenho avaliados foram o ganho médio de peso diário (GMD), ganho médio de peso no período, peso corporal final e, como indicadores econômicos, a receita total, custo operacional total por arroba (COT/@), margem bruta e margem líquida. O GMD na recria foi semelhante $(\mathrm{P}>0,05)$ entre os grupos que apresentaram média de 746 e 456 gramas, respectivamente, para os períodos águas/transição águasseca e seca. O COT/@ da recria do grupo Tricross $(\mathrm{R} \$ 69,44)$ foi superior aos dos grupos Guzonel (R\$66,75) e Guzolando (R\$ 64,86). No período de terminação, o GMD foi semelhante entre os grupamentos, apresentanto o valor médio de 1,55kg. O COT/@ da terminação em confinamento foi R\$ 91,48, R \$114,15 e R \$122,78, para Tricross, Guzonel e Guzolando, respectivamente. Considerando-se o sistema de ciclo curto completo, obteve-se COT/@ menor para o grupamento Tricross $(\mathrm{R} \$ 77,92)$, seguido pelo grupamento Guzonel (R\$ 74,89) e Guzolando (R\$ 78,43). Nas condições de produção avaliadas, o grupo Tricross foi o mais eficiente economicamente.

Palavras-chave: custo de produção, ganho de peso, sistema de produção, lucro

\begin{abstract}
The productive and economic performance of different beef genetic groups in a pasture-based growing system and feedlot finishing with one year cycle was evaluated. Thirty-six bulls from three groups F1Guzerat x Nellore F1 (GN); F1 Guzerá x Hosltein (GH) and F1 1/2 Simmental $+1 / 4$ Guzerá + 1/4 Nellore $(S G)$, with average age of 270 ( \pm 30$)$ days and average body weight of $241.3( \pm 34.0) \mathrm{kg}$ were utilized. The experimental design utilized was completely randomized $(3 \times 12)$. During the growing phase, bulls were kept in rotational Brachiaria brizantha $c v$ Marandu pastures, receiving commercial supplement; in the finishing phase, they received a diet containing sorghum silage and concentrate (50:50) for 61 days. The performance indices evaluated were average daily weight gain (ADG), average daily gain during the period (ADP), final body weight. Economic performance indicators were total revenue, total operating expenses per bushel (COT / @), gross margin and net margin. Average daily gain during the growing phase was similar $(P>0.05)$ among groups with an average of 746 and 456 grams, respectively, for the
\end{abstract}

Recebido em 25 de julho de 2013

Aceito em 3 de outubro de 2013

E-mail: felipemoreira@zootecnista.com.br 
period's transitional rainy / dry season. The TOE/bushel recreates the grouping of $S D$ (R\$ 69.44) was higher than that of groups $G N(R \$ 66.75)$ and $G H(R \$ 64.86)$. In the finishing period, $A D G$ was similar $(P>0.05)$ among groups with an average of $1.55 \mathrm{~kg}$. The TOE/bushel of the feedlot finishing were $R \$$ $91.48, R \$ 114.15$ and $R \$ 122.78$ respectively for $S D, G N$ and GH groups. Considering the analysis of the short-cycle system, we obtained the lowest TOE/bushel for grouping $S D$ (R $\$ 77.92)$ followed by grouping $G N(R \$ 74.89)$ and $G H(R \$ 78.43)$. These results provided higher profitability for the crosses $S D$ (21.99\%) compared to other intersections (18.92\% and $18.31 \%$ respectively, and for GN and GH). In the conditions evaluated the $S D$ group was the most economically efficient.

Keywords: production cost, production system, operating income, weight gain

\section{INTRODUÇÃO}

A cadeia produtiva da carne bovina passou, nos últimos anos, a incorporar tecnologias que visam à melhoria da eficiência e aumento da produção impostas pela globalização e competitividade, frente a outras cadeias de proteína animal. A pecuária extensiva a pasto contribui para o saldo positivo do comércio de bovinos no Brasil, entretanto seu maior tempo de ciclo produtivo ocasiona perdas econômicas, o que minimiza seu resultado financeiro final.

Segundo Oaigen et al. (2008), a redução da margem de lucro, o aumento da concorrência e as exigências por produção de carne de melhor qualidade e de menor custo tornaram a pecuária de ciclo longo, com baixas produtividade e qualidade de produto, economicamente inviável. Assim, estratégias como a suplementação múltipla, especialmente a proteica ao longo do ano, bem como a terminação em confinamento, surgem como opção para aumentar a eficiência do sistema e reduzir o ciclo de produção. Em muitas regiões, tais práticas são difundidas na produção de bovinos de corte, contribuindo assim para aumentar a produção de arrobas por hectare.

As estratégias para maior desempenho produtivo devem também ser acompanhadas com melhor resultado financeiro. Nesse sentido, a análise econômica da atividade pecuária é extremamente importante, fundamental para utilizar, de maneira inteligente e econômica, os fatores de produção (Lopes e Carvalho, 2002).

A utilização do cruzamento entre zebuínos e taurinos na região do cerrado brasileiro tem grande potencial de produzir animais com crescimento adequado a pasto e terminação rápida em confinamento com alta qualidade de carne. A região de Curvelo, Minas Gerais, tem disponibilidade de bovinos da raça Guzerá, bem como de seus cruzamentos voltados tanto para o corte quanto para o leite. Utilizar recursos genéticos adaptados à região pode ser opção estratégica para produção de animais de ciclo curto, compatíveis com características de desempenho de carcaça desejável pela pecuária moderna.

Objetivou-se, no presente estudo, avaliar o desempenho produtivo de bovinos de três grupamentos genéticos, quanto a seu desenvolvimento ponderal, nas fases de recria e terminação, além do desempenho econômico dos grupos nesse sistema de ciclo curto de produção.

\section{MATERIAL E MÉTODOS}

O estudo foi conduzido no município de Curvelo, região central de Minas Gerais, no período entre 9 de novembro de 2011 a 12 de novembro de 2012. As avaliações foram divididas em duas fases: a recria a pasto, realizada na Fazenda Canoas (latitude $18^{\circ} 44^{\prime} 57^{\prime \prime} \mathrm{S}, 44^{\circ} 26^{\prime} 48^{\prime \prime} \mathrm{O}$ ); e a terminação, no confinamento da Fazenda Experimental do Moura, da Universidade Federal dos Vales Jequitinhonha e Mucuri (latitude $18^{\circ} 49^{\prime} 54.73$ "S e longitude $44^{\circ} 23^{\prime} 39.43^{\prime \prime} \mathrm{O}$ ).

Foram utilizados 36 bovinos, inteiros, de três grupamentos genéticos com base na raça Guzerá, sendo doze Guzonel (F1Guzerá $x$ Nelore), doze Guzolando (F1Guzerá $x$ Holandês) e doze Tricross ( $1 / 2$ Guzonel $x \quad 1 / 2$ Simental), com idade média de oito meses $( \pm 1)$, oriundos do mesmo rebanho. O peso inicial médio foi de $277 \pm 33 \mathrm{~kg}$; $259 \pm 27 \mathrm{~kg}$ e $207 \pm 4 \mathrm{~kg}$ para os grupos Tricross, Guzonel e Guzolando, respectivamente. Os animais foram manejados no mesmo lote, em pastagem de Brachiaria brizantha cv. Marandu (Brachiarão) não adubado, rotacionados em piquetes com média de trinta hectares e com taxa de ocupação de uma unidade animal por hectare 
(1UA/ha). Os animais receberam suplementos comerciais, conforme recomendação da empresa fornecedora, dividindo-se a recria entre fases, conforme a pluviometria regional. A primeira fase compreendeu a época das águas e transição águas/seca, utilizando-se dois suplementos, com consumo estimado de $0,04 \%$ e $0,08 \%$ do peso vivo por dia e níveis de garantia (por quilograma de suplemento) de $160 \mathrm{~g}$ de proteína bruta (PB); $55 \mathrm{~g}$ de fósforo (P); $77 \mathrm{~g}$ de sódio; $60 \mathrm{~g}$ de nitrogênio não proteico equivalente proteico (NNP) e $300 \mathrm{~g}$ de $\mathrm{PB} ; 18 \mathrm{~g}$ de fósforo; $81 \mathrm{~g}$ de sódio; $190 \mathrm{NNP}$, respectivamente. A segunda fase compreendeu parte da estação seca na região, utilizando-se suplemento com consumo estimado de $0,1 \%$ do peso corporal por dia e níveis de garantia por $\mathrm{kg}$ de suplemento de $400 \mathrm{~g}$ de PB; $18 \mathrm{~g}$ de P; $115 \mathrm{~g}$ Na e $340 \mathrm{~g}$ de NNP.

O confinamento foi composto por 12 currais e, para cada um deles, foram sorteados três animais do mesmo grupo genético (área de $120 \mathrm{~m}^{2}$, sem cobertura ou piso pavimentado, com comedouro de cimento pré-moldado coberto e bebedouros individuais regulados por torneira boia). Os animais foram adaptados ao manejo alimentar e às instalações por período de 22 dias. A dieta de adaptação consistiu em silagem de sorgo e concentrado (o mesmo utilizado no confinamento) na relação inicial V:C (90:10), com base na matéria seca (MS). Gradativamente aumentou-se a participação do concentrado na dieta até apresentar relação V:C de 50:50, igual à dieta experimental. Após a adaptação, os animais foram submetidos a 61 dias de avaliação do desempenho. $\mathrm{O}$ concentrado foi composto por milho quebrado, sorgo moído, farelo de soja, farelo de algodão, ureia e núcleo mineral $(88,9$ $\% \mathrm{MS} ; 16,02 \% \mathrm{~PB}$ e $13,26 \%$ FDN) e o volumoso utilizado foi a silagem de sorgo $(37,7 \% \mathrm{MS}$; $6,38 \%$ PB; 52,48\% FDN).

A dieta foi fornecida duas vezes ao dia: uma metade pela manhã (às 8 horas) e a outra metade à tarde (às 15 horas). O consumo diário foi ajustado de acordo com as sobras do cocho (5\% do oferecido).

O consumo de matéria seca (CMS) foi determinado em cada curral através da diferença dos pesos $(\mathrm{kg})$ da dieta fornecida e da sobra alimentar, multiplicados pelos respectivos valores de MS.
Para avaliação do desempenho produtivo, foram coletados dados de nove pesagens individuais, em jejum, sendo cinco na fase de recria, com intervalos de três meses, e quatro pesagens na terminação, com intervalo de 21 dias. Determinaram-se o ganho de peso médio diário (GMD) e ganho de peso total (GPT) nos dois períodos experimentais, além do ganho de peso em carcaça (GPC) e rendimento médio de carcaça quente (RCQ) após o abate dos animais. Este último índice, RCQ, foi utilizado para estimar o ganho em arrobas no confinamento, fundamental para medir a economicidade dos grupamentos no sistema de produção.

O delineamento experimental utilizado foi $\mathrm{o}$ inteiramente casualizado. As variáveis foram analisadas pelo procedimento GLM (General Linear Model) do SAS (Statistical..., 2002), considerando-se o peso corporal inicial como covariável. As médias foram comparadas pelo teste de Tukey, utilizando-se 5\% de significância. O modelo estatístico utilizado foi o seguinte:

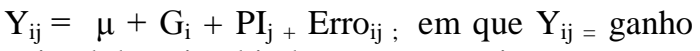
animal do animal $\mathrm{j}$, do grupamento $\mathrm{i}$;

$\mu=$ média do efeito; $\mathrm{G}_{\mathrm{i}}=$ grupamento genético $(\mathrm{i}=0,1,2) ; \mathrm{PI}_{\mathrm{j}}=$ peso inicial do animal $\mathrm{j}$; e Erro $_{\mathrm{ij}}$ $=$ erro aleatório associado a cada animal.

Para avaliação do desempenho econômico, utilizou-se a metodologia proposta por Matsunaga et al. (1976). Os custos da recria e do confinamento tiveram como base os coeficientes técnicos apontados pelo Anualpec (Anuário..., 2012), corrigidos pelo IGP-DI da Fundação Getúlio Vargas para dezembro de 2012. Utilizaram-se os seguintes valores já corrigidos, por animal e por ano: sanidade, $\mathrm{R} \$ 6,94$; manutenção de pastagens, R\$ 15,87; manutenção de instalações, $\mathrm{R} \$ 26,55$; manutenção de equipamentos, $\mathrm{R} \$ 11,93$; despesas gerais e administrativas, $\mathrm{R} \$ 11,25$; e energia, $\mathrm{R} \$ 1,30$. O custo com mão de obra foi alcançado pela relação de 393 bois por homem, conforme indica o Anualpec (Anuário..., 2012), com salário-base na região no valor de $\mathrm{R} \$ 1.000,00$.

Para o confinamento, as despesas com a dietas foram obtidas com base nos preços regionais. $\mathrm{E}$ os demais foram: mecanização, R \$ 6,90; mão de obra e encargos, R\$10,33; medicamentos, R \$ 12,47; despesas administrativas, $\mathrm{R} \$ 7,46$; e 
depreciações, $R$ \$ 3,90, todos também por animal e por ano.

O valor de aquisição dos animais na recria foi calculado a partir do peso médio dos animais, a partir da cotação da arroba (@) praticada pelo mercado regional em novembro de 2011 (R\$ 95,00/@), sendo R\$ 875,00, R\$ 780,00 e R\$ 680,00 para Tricross, Guzonel e Guzolando, respectivamente. $\mathrm{O}$ custo com a suplementação foi calculado através da multiplicação do consumo de cada grupo pelo custo regional do suplemento.

Para avaliação do ciclo todo (recria e engorda), a determinação do custo de aquisição dos animais no confinamento, foi utilizado o custo operacional total da recria, partindo da prerrogativa de que no sistema as duas fases são complementares. Entretanto, na avaliação da atividade confinamento, utilizaram-se o peso médio dos animais ao final da recria e a cotação da arroba praticada pelo mercado regional para essa categoria ( $\mathrm{R} \$ 89,00)$, o que resultou nos seguintes valores: $\mathrm{R} \$ 1.300,00, \mathrm{R} \$ 1.260,00 \mathrm{e}$ 1.130,00 para Tricross, Guzonel e Guzolando, respectivamente.
A receita total (RT) na recria foi simulada através da venda dos animais no mês de agosto de 2012, utilizando-se o peso dos mesmos versus o preço da arroba do boi gordo na época $(\mathrm{R} \$$ 83,00/@). Para a RT do sistema integrado, foi utilizada a mesma metodologia (R\$ 96,00/@, em 12 de novembro de 2012).

A partir dos custos de produção e das receitas, foram obtidos os indicadores econômicos margem bruta (MB), margem líquida (ML) e taxa interna de retorno (TIR). Foram apresentadas análises de cada fase do sistema de produção e, posteriormente, a análise total de todo o ciclo como resultado final.

\section{RESULTADOS E DISCUSSÃO}

Não houve diferença $(\mathrm{P}>0,05)$ no desempenho produtivo entre os grupos genéticos durante $\mathrm{o}$ período das águas e transição águas/seca da fase de recria (Tab. 1); tendo sido observados valores médios para GMD nas respectivas fases de $771,7 \mathrm{~g} /$ dia e $447,9 \mathrm{~g} /$ dia.

Tabela 1. Desempenho de bovinos de três grupos genéticos na fase de recria em pasto durante os períodos das águas, transição de águas/seca e seca

\begin{tabular}{lccccc}
\hline \multicolumn{1}{c}{ Variáveis } & Tricross & Guzonel & Guzolando & Valor de $\mathrm{P}^{1}$ & CV (\%) \\
\hline \multirow{5}{*}{ Peso inicial $(\mathrm{kg})$} & $275,2( \pm 20)$ & $259,5( \pm 27)$ & $207,4( \pm 14)$ & \\
Peso final $(\mathrm{kg})$ & $404,8( \pm 27)$ & $374,6( \pm 38)$ & $323,6( \pm 22)$ & 0,629 & 8,29 \\
GMD $^{2}(\mathrm{~g})$ & $822,5( \pm 0,10)$ & $742,9( \pm 0,08)$ & $749,8( \pm 0,13)$ & 0,962 & 25,44 \\
& $( \pm 0,10)$ & $( \pm 0,08)$ & Período da seca \\
Peso inicial $(\mathrm{kg})(\mathrm{kg})$ & $404,8( \pm 27)$ & $374,6( \pm 38)$ & $323,6( \pm 22)$ & 0,629 & 8,29 \\
Peso final $(\mathrm{kg})$ & $458,4^{\mathrm{a}}( \pm 30)$ & $430,1^{\mathrm{b}}( \pm 42)$ & $388,0^{\mathrm{c}}( \pm 24)$ & 0,002 & 2,51 \\
GMD $^{2}(\mathrm{~g})$ & $415,3( \pm 0,05)$ & $429,5( \pm 0,06)$ & $498,8( \pm 0,07)$ & 0,848 & 18,54 \\
\hline
\end{tabular}

${ }^{1}$ Médias na mesma linha seguidas de letras diferentes se diferem pelo teste Tukey $(\mathrm{P}<0,05)$. ${ }^{2} \mathrm{GMD}$ : ganho médio diário.

Esses resultados podem ser considerados satisfatórios para desempenho de animais a pasto. Provavelmente, as condições de recria nesse período favoreceram de maneira semelhante os animais de todos os grupos. Ganhos de peso semelhantes (746 g/dia) foram relatados por Barbosa et al. (2008) na recria de novilhos cruzados recebendo suplementos com consumo de $0,37 \%$ do peso corporal no período de transição águas-seca.
Já para o período da seca da recria houve diferença $(\mathrm{P}<0,05)$ no $\mathrm{PCF}$, sendo que o grupo Tricross apresentou maior peso $(458,4 \mathrm{~kg})$ que os demais grupos e, entre estes, o Guzonel foi superior $(430,1 \mathrm{~kg})$ ao Guzolando $(388,0 \mathrm{~kg})$. Entretanto, não houve efeito $(\mathrm{P}>0,05)$ no GMD, que apresentou média 447,8g/dia.

A diferença entre os grupos genéticos no PCF no período da seca, provavelmente, ocorreu devido à diferença numérica do peso corporal inicial, 
mesmo este sendo analisado como co-variável. Entretanto, o GMD semelhante entre os grupamentos é a variável mais importante e isso demonstra o mesmo potencial de ganho, como ocorrido no período das águas e transição águas/seca.

O GMD obtido no período da seca foi próximo ao valor médio encontrado por Porto et al. (2011) na recria com suplementação de bovinos Nelore (483g/dia), e por Figueiredo et al. (2008), ao testarem diferentes estratégias de suplementação múltipla na recria com animais Holandês $x$ Zebu (480g/dia).

Quando considerada toda a fase de recria, observou-se GMD de 626g/dia, semelhante $(\mathrm{P}>0,05)$ entre os três grupos genéticos. Além disso, o GMD apresentado nessa fase pode ser considerado satisfatório para o sistema que preconiza o ciclo curto na bovinocultura de corte.

Segundo Reis et al. (2009), com a suplementação múltipla como estratégia de manejo a pasto, ganhos médios de $500 \mathrm{~g}$ a $600 \mathrm{~g}$ por dia são fundamentais para que animais sejam abatidos na faixa dos vinte meses de idade.

Sobre os resultados econômicos da fase de recria, os animais Tricross apresentaram maior RT que os demais grupos, devido ao maior peso corporal e, consequentemente, à maior quantidade de arrobas vendidas (Tab. 2). Entretanto, o COT e o COT por arroba desse grupamento ( $\mathrm{R} \$ 1.055,52$ e $\mathrm{R} \$ 69,44$, respectivamente) também foram superiores aos dos Guzonel (R\$ 954,48 e R $\$ 66,75)$ e Guzolando ( $\mathrm{R} \$ 836,64$ e $\mathrm{R} \$$ 64,86), o que resultou em menor margem líquida (ML) no período de recria.

Tabela 2. Análise econômica (por animal) da recria em pasto de bovinos de três grupos genéticos

\begin{tabular}{lccc}
\hline Variáveis & Tricross & Guzonel & Guzolando \\
\hline Receita Total (R\$) & $1.261,6$ & $1.186,9$ & $1.070,7$ \\
Arrobas vendidas & 15,20 & 14,30 & 12,90 \\
Custo Operacional Total (R\$) & $1.055,52$ & 954,48 & 836,64 \\
Custo Operacional Efetivo (R\$) & $1.039,91$ & 938,87 & 821,03 \\
Margem Bruta (R\$) & 206,08 & 232,42 & 234,06 \\
Margem Líquida (R\$) & 221,69 & 248,03 & 249,67 \\
COT/@ ${ }^{1}(\mathrm{R} \$)$ & 69,44 & 66,75 & 64,86 \\
\hline
\end{tabular}

${ }^{1} \mathrm{COT} / @$ : custo operacional total por arrobas vendidas.

Os animais Guzolando propiciaram margem líquida maior que os animais Tricross e Guzonel, respectivamente, em $\mathrm{R} \$ 27,98$ $(12,62 \%)$ e $\mathrm{R} \$ 1,64(0,66 \%)$ por animal. Observou-se, porém, que todos os grupos apresentaram ML positiva na fase de recria. Esse resultado indicou que a fase de recria desses animais seria viável em longo prazo.

A menor ML referente à comercialização dos animais Tricross durante o período de recria pode ser explicada pelo seu maior custo de aquisição. A aquisição dos animais nessa fase propiciou o maior desembolso, como já esperado, acima de $80 \%$ do custo operacional efetivo (COE) em todos os grupos genéticos. Entretanto, os animais Tricross apresentaram peso inicial à compra superior em $67,8 \mathrm{~kg}$ e $15,7 \mathrm{~kg}$ em relação aos animais Guzolando e Guzonel, respectivamente, o que teve grande impacto no COT por arroba vendida após a recria. O resultado demonstrou a importância que a compra de animais de reposição exerce no COE e COT dentro da bovinocultura de corte. Além disso, foi observado que o maior PCF e, consequentemente, a maior quantidade de arrobas vendidas dos animais Tricross não compensaram seu maior custo de aquisição.

O custo da suplementação correspondeu, na média, a 8,37\% do COE no período de recria. Esse resultado difere dos apresentados por Figueiredo et al. (2007) e Barbosa et al. (2008), que relataram média de $20 \%$ e $25 \%$ do desembolso com suplementação na recria, respectivamente. Entretanto, os autores não contabilizaram o custo de aquisição de animais, o que contribuiu para aumentar a participação da 
suplementação no COE. O custo da suplementação na recria pode ser influenciado pelo ano de estudo e época de compra de insumos, e essas variações podem levar a diferentes resultados.

$\mathrm{Na}$ fase do confinamento, não houve diferença $(\mathrm{P}>0,05)$ no GMD e no GPT entre os grupamentos que apresentaram média de $1,53 \mathrm{~kg} / \mathrm{dia}$ e $95,45 \mathrm{~kg}$, respectivamente (Tab. 3). Entretanto, houve diferença $(\mathrm{P}<0,05)$ no $\mathrm{PCF}$ e no GPC, sendo que o grupamento Tricross apresentou valores de, respectivamente, $559,0 \mathrm{~kg}$ e 79,7kg, os quais foram superiores aos dos demais grupamentos.

Já para o RCQ, os grupos Tricross e Guzonel foram semelhantes $(\mathrm{P}<0,05)$, porém superiores ao grupo Guzolando $(\mathrm{P}<0,05)$. Houve diferença $(\mathrm{P}<0,05)$ no consumo de matéria seca $(\mathrm{CMS})$ em relação ao peso corporal (CMS/PC) entre os cruzamentos, sendo que os animais Guzolando apresentaram o maior consumo $(2,77 \%$ do PC), seguidos pelos animais Guzonel (2,58\% do PC) e Tricross $(2,40 \%$ do PC). Entretanto, a conversão alimentar (CA) foi semelhante $(\mathrm{P}>0,05)$ entre os grupos genéticos, com média de 8,02.

Tabela 3. Desempenho produtivo de bovinos de três grupos genéticos em confinamento

\begin{tabular}{lccccc}
\hline Variáveis & Tricross & Guzonel & Guzolando & Valor de $\mathrm{P}^{1}$ & CV $(\%)$ \\
\hline Peso inicial $(\mathrm{kg})$ & $461,6( \pm 11)$ & $435,1( \pm 20)$ & $395,3( \pm 14)$ & & \\
Peso final $(\mathrm{kg})$ & $559,05 \mathrm{a}( \pm 15)$ & $520,9 \mathrm{~b}( \pm 16)$ & $498,27 \mathrm{c}( \pm 12)$ & $<0,001$ & 1,00 \\
$\mathrm{GMD}^{2}(\mathrm{~kg})$ & $1,56( \pm 0,12)$ & $1,40( \pm 0,12)$ & $1,68( \pm 0,04)$ & 0,253 & 5,53 \\
$\mathrm{GPT}^{3}(\mathrm{~kg})$ & $97,50( \pm 4,9)$ & $85,82( \pm 7,5)$ & $103,07( \pm 2,7)$ & 0,253 & 5,53 \\
$\mathrm{GPC}^{4}(\mathrm{~kg})$ & $79,79 \mathrm{a}$ & $62,62 \mathrm{~b}$ & $59,41 \mathrm{~b}$ & 0,025 & 10,37 \\
$\mathrm{RCQ}^{5}(\%)$ & $56,03 \mathrm{a}( \pm 1,8)$ & $54,23 \mathrm{a}( \pm 1,2)$ & $51,56 \mathrm{~b}( \pm 1,5)$ & 0,004 & 2,38 \\
$\mathrm{CMS}^{6}(\mathrm{~kg} / \mathrm{dia})$ & 12,56 & 12,37 & 12,41 & 0,057 & 1,07 \\
$\mathrm{CMS}^{\mathrm{P}} \mathrm{PC}^{7}(\%)$ & $2,46 \mathrm{c}$ & $2,58 \mathrm{~b}$ & $2,77 \mathrm{a}$ & 0,003 & 1,24 \\
$\mathrm{CA}^{8}$ & 7,87 & 8,85 & 7,35 & 0,096 & 6,79 \\
\hline
\end{tabular}

${ }^{1}$ Médias na mesma linha seguidas de letras diferentes se diferem pelo teste Tukey $(\mathrm{P}<0,05) .{ }^{2} \mathrm{GMD}$ : ganho médio diário. ${ }^{3} \mathrm{GPT}$ : ganho de peso total. ${ }^{4} \mathrm{GPC}$ : ganho de peso em carcaça total. ${ }^{5} \mathrm{RCQ}$ : rendimento carcaça quente. ${ }^{6} \mathrm{CMS}$ : consumo matéria seca. ${ }^{7} \mathrm{CMS} / \mathrm{PC}$ : consumo matéria seca em relação ao peso corporal. ${ }^{8} \mathrm{CA}$ : conversão alimentar.

Os resultados de desempenho demonstraram que os animais Tricross foram mais eficientes em produzir carcaça, mesmo apresentando GMD e CA semelhantes aos dos demais cruzamentos. Provavelmente, a heterose propiciada pelos cruzamentos entre Bos taurus indicus (Nelore e Guzerá) e Bos taurus (Simental) contribuiu para esses resultados, já que são raças selecionadas para a produção de carne. Por outro lado, os animais Guzolando tiveram maior participação de raça taurina (Holandês) selecionada para produção de leite. A literatura reporta maior participação de órgãos e gordura visceral no ganho de peso de corpo vazio de animais F1 Holandês $x$ Zebu comparado a cruzamentos entre taurinos e zebuínos selecionados para corte (Fernandes et al., 2005). Já para os animais Guzonel, a heterose propiciada entre o cruzamento de raças zebuínas (Guzerá e Nelore) resultou em características de desempenho intermediárias em relação aos demais cruzamentos. Os resultados observados para o RCQ corroboram as afirmações acima e indicam maior eficiência do grupo Tricross em relação ao Guzolando.

Entretanto, o cruzamento Tricross apresentou menor COT por arroba produzida no confinamento ( $\mathrm{R} \$ 91,48$ ) comparado ao dos demais cruzamentos ( $\mathrm{R} \$ 114,15$ e $\mathrm{R} \$ 122,78$ para Guzonel e Guzolando, respectivamente) (Tab. 4). Ressalta-se que o COT das arrobas produzidas no confinamento pelos grupos Guzonel e Guzolando foram superiores ao preço da arroba para venda, e ocasionaram deficit por arroba de $\mathrm{R} \$ 18,15$ e $\mathrm{R} \$ 16,78$, respectivamente. Esse resultado demonstrou a importância de estimativas dos custos de produção para o planejamento adequado do confinamento. O custo com alimentação no confinamento foi, em média, 27,56\% do COT; quando desconsiderado o custo de aquisição dos animais, sua significância passa para, em média, 94,15\%. Em outros estudos, como o de Coan et al. (2008) e Pacheco et al. (2006), o custo com alimentação (volumoso mais concentrado) foi, aproximadamente, de $85 \%$ e $75,54 \%$ do COT, 
respectivamente, desconsiderando-se o custo de aquisição. Diferenças no preço de compra de insumos, na relação volumoso:concentrado e no peso corporal dos animais, podem explicar a variação percentual da alimentação no COT.

Tabela 4. Análise econômica da terminação em confinamento de bovinos de três grupos genéticos

\begin{tabular}{lccc}
\hline Variáveis & Tricross & Guzonel & Guzolando \\
\hline Receita Total $(\mathrm{R} \$)$ & $2.004,48$ & $1.803,84$ & $1.644,48$ \\
Custo Operacional Total $(\mathrm{R} \$)_{\text {Custo Operacional Efetivo }^{1}(\mathrm{R} \$)}^{1.819,60}$ & $1.808,24$ & $1.779,37$ & $1.648,14$ \\
Margem Bruta $(\mathrm{R} \$)^{\text {Margem Liquida }(\mathrm{R} \$)}$ & 196,24 & $1.768,01$ & $1.636,79$ \\
$\mathrm{COT} / @$ produzidas $^{2,3}(\mathrm{R} \$)$ & 184,88 & 35,83 & 7,69 \\
Diária confinamento $^{4}(\mathrm{R} \$)$ & 91,48 & 24,47 & $-3,66$ \\
\hline
\end{tabular}

Incluindo o custo de aquisição dos animais. ${ }^{2} \mathrm{COT} / @$ produzidas: custo operacional total por arrobas produzidas no confinamento. ${ }^{3}$ Não incluindo a aquisição dos animais. ${ }^{4}$ Custo operacional total por dia sem o custo de aquisição dos animais.

Pode ser observado também através da ML que o confinamento do grupo Guzolando foi inviável se analisado isoladamente. Também Lopes et al. (2011), ao avaliarem a viabilidade econômica da terminação em confinamento de novilhos Nelore e Red Norte no ano de 2007, obtiveram resultados negativos, indicando que a atividade apresentou prejuízo. Por outro lado, Ferreira et al. (2009) trabalharam com diferentes grupos genéticos e apresentaram resultados positivos para todos os grupos estudados. Da mesma forma que Lopes e Magalhães (2005), que avaliaram os resultados econômicos de um confinamento de 3.583 animais no ano de 2002, e observaram resultados positivos, levando à conclusão de que a atividade teria condições de sobreviver no longo prazo.

O valor observado para a diária no confinamento foi semelhante entre os grupamentos e foi em média R\$ 6,25. Esse índice é comumente utilizado em meio aos confinadores e indica os custos diários (alimentação, mão de obra, combustível, despesas gerais etc.) do confinamento por boi, não incluindo o custo com aquisição dos animais.

Quando analisado o período experimental completo (recria e confinamento), observou-se diferença $(\mathrm{P}<0,05)$ apenas para a variável $\mathrm{PCF}$ (Tab. 5), o que foi devido às diferenças nos pesos iniciais. Entretanto, não foram observadas diferenças (P>0,05) no GMD, GPT e GMC entre os grupos, que apresentaram média de $755 \mathrm{~g}$, 8,93@ e 9,52@, respectivamente. Tais resultados demonstraram que os três grupos genéticos apresentaram desempenho produtivo satisfatório no sistema de ciclo curto.

Tabela 5. Desempenho produtivo de bovinos de três grupos genéticos em sistema de ciclo curto de produção

\begin{tabular}{lccccc}
\multicolumn{1}{c}{ Variáveis } & Tricross & Guzonel & Guzolando & Valor de $\mathrm{P}^{1}$ & CV $(\%)$ \\
\hline Peso inicial $(\mathrm{kg})$ & 275,20 & 259,50 & 207,40 & & \\
Peso final $(\mathrm{kg})$ & $559,10^{\mathrm{a}}$ & $520,90^{\mathrm{b}}$ & $498,30^{\mathrm{c}}$ & $<0,001$ & 3,70 \\
$\mathrm{GMD}^{2}(\mathrm{~g})$ & 765,60 & 710,50 & 790,60 & 0,243 & 7,00 \\
$\mathrm{GPT}^{3}(@)$ & 9,03 & 8,42 & 9,34 & 0,487 & 7,28 \\
$\mathrm{GMC}^{4}(@)$ & 9,79 & 8,97 & 9,81 & 0,292 & 7,14 \\
\hline
\end{tabular}

${ }^{1}$ Médias na mesma linha seguidas de letras diferentes se diferem pelo teste Tukey $(\mathrm{P}<0,05) ;{ }^{2} \mathrm{GMD}$ : ganho médio diário. ${ }^{3} \mathrm{GPT}$ : ganho de peso total por arrobas. ${ }^{4} \mathrm{GMC}$ : ganho médio de carcaça em arrobas.

Observou-se que o COT/@ ao final do ciclo entre os três cruzamentos foram próximos, apresentando média de $\mathrm{R} \$ 77,65$ (Tab. 6). Ainda assim, a análise econômica de todo o período experimental demonstrou que o grupo Tricross propiciou maior margem líquida ( $\mathrm{R} \$ 440,71)$, seguido pelo grupo Guzonel ( $\mathrm{R} \$ 341,35)$ e Guzolando (R\$ 301,05). O maior RCQ (Tabela 3) do grupo Tricross e, consequentemente, maior quantidade de arrobas vendidas (20,88@), 
compensaram o maior COT ( $\mathrm{R} \$ 1.563,77)$, resultando em maior ML.

$\mathrm{Na}$ fase de recria, o grupo Guzolando foi o mais eficiente, pois apresentou menor COT
(Tab. 2); entretanto, o pior RCQ (Tab. 3) resultou em menor quantidade de arrobas vendidas, reduzindo a ML na análise final de todo o ciclo curto. Já para o grupo Tricross, observou-se maior MB e ML ao final do ciclo.

Tabela 6. Análise econômica de bovinos de três grupos genéticos de todo o período experimental (por animal)

\begin{tabular}{lccc}
\hline Variáveis & Tricross & Guzonel & Guzolando \\
\hline Receita Total (R\$) & $2.004,48$ & $1.803,84$ & $1.644,48$ \\
Arrobas produzidas (@) & 20,88 & 18,79 & 17,13 \\
Custo Operacional Total (R\$) & $1.575,12$ & $1.473,85$ & $1.354,78$ \\
Custo Operacional Efetivo (R\$) & $1.563,77$ & $1.462,49$ & $1.343,43$ \\
Custo Operacional Total/@ (R\$) & 74,89 & 77,83 & 78,43 \\
Margem Bruta (R\$) & 440,71 & 341,35 & 301,05 \\
Margem Líquida (R\$) & 429,36 & 329,99 & 289,70 \\
TIR $^{1}$ & $5,41 \%$ & $1,40 \%$ & $-0,19 \%$ \\
\hline
\end{tabular}

${ }^{\mathrm{I}}$ TIR: taxa interna de retorno.

Observou-se que os todos os animais apresentaram ML positiva. O destaque foi para os animais Tricross, que apresentaram ML superior à dos animais Guzolando e Guzonel. A grande diferença demonstra o potencial desse cruzamento no sistema de ciclo curto de produção.

O COT por arroba, no sistema, entre todos os grupos genéticos, foi inferior ao preço de comercialização ( $\mathrm{R} \$ 96,00)$. Portanto, no final desse ciclo de produção, os três grupos apresentaram ML positiva; sendo assim, considerados viáveis em médio e longo prazo. É importante ressaltar que, mesmo a atividade do confinamento podendo não ser viável de forma isolada, fazendo parte do sistema de produção pode ser uma estratégia interessante, em especial se forem consideradas outras vantagens dessa atividade, como liberação de pastagem e garantia de produto na entressafra.

Considerando um fluxo de caixa de quinze anos, os animais Tricross apresentariam taxa interna de retorno de $5,41 \%, 3,8$ vezes superior à apresentada pelo grupo Guzonel. $\mathrm{O}$ grupo Guzolando, com esse prazo, indica uma TIR de $0,19 \%$, indicando que, nesse grupo, o retorno financeiro foi praticamente nulo, podendo ser considerado de maior risco.

\section{CONCLUSÃO}

O sistema de ciclo curto no município de Curvelo, Minas Gerais, para os três grupos genéticos, apresenta desempenho produtivo eficiente, além de ser viável economicamente. Entretanto, os animais do grupo Tricross foram mais eficientes economicamente nas condições de produção avaliada.

\section{REFERÊNCIAS}

ANUÁRIO da pecuária brasileira-Anualpec. São Paulo: FNP Consultoria \& Comércio, 2012. 385p.

BARBOSA, F.A; GRAÇA, D.S; GUIMARÃES, P.H.S. et al. Análise econômica da suplementação proteico-energética de novilhos durante o período de transição entre água-seca. Arq. Bras. Med. Vet. Zootec., v.60, p.911-916, 2008.

COAN, R.M.; REIS, R.A.; RESENDE, F.D. et al. Viabilidade econômica, desempenho e características de carcaça de garrotes em confinamento alimentados com dietas contendo silagem de capins tanzânia ou marandu ou silagem de milho. Rev. Bras. Zootec., v.37, p.311-318, 2008. 
FERNANDES, H.J.; PAULINO, M.F.; MARTINS, R.G.R. et al. Crescimento de componentes corporais de três grupos genéticos nas fases de recria e terminação. Rev. Bras. Zootec., v. 34, p.288-296, 2005.

FERREIRA, I.C.; SILVA, M.A.; BARABOSA, F.A. et al. Avaliação técnica e econômica de diferentes grupos genéticos de bovinos de corte machos superprecoces e do sistema de produção em confinamento. Arq. Bras. Med. Vet. Zootec., v.61, p.243-250, 2009.

FIGUEIREDO, D.M.; OLIVEIRA, A.S.; SALES, M.F.L. et al. Análise econômica de quatro estratégias de suplementação para recria e engorda de bovinos em sistema pastosuplemento. Rev. Bras. Zootec., v.36, p.14431453, 2007.

FIGUEIREDO, D.M.; PAULINO, M.F.; DETMANN, E. et al. Fontes de proteína em suplementos múltiplos para bovinos em pastejo no período das águas. Rev. Bras. Zootec., v.37, p.2222-2232, 2008.

LOPES, M.A.; CARVALHO, F.M. Custo de Produção de Gado de Corte. Lavras: UFLA. 2002. 47p. (Boletim Agropecuário, 47).

LOPES, M.A.; MAGALHÃES, G.P. Análise da rentabilidade da terminação de bovinos de corte em condições de confinamento: um estudo de caso. Arq. Bras. Med. Vet. Zootec., v.57, p.374$379,2005$.
LOPES, S.M.; LADEIRA, M.M.; MACHADO NETO, O.R. et al. Viabilidade econômica da terminação de novilhos Nelore e Red Norte em confinamento na região de Lavras-MG. Cienc. Agrotec., v.35, p.774-780, 2011.

MATSUNAGA, M.; BEMELMANS, P.F.; TOLEDO, P.E.N. et al. A. Metodologia de custo de produção utilizado pelo IEA. Agric. em São Paulo, v.23, p.123-139, 1976.

OAIGEN, R.P.; BARCELLOS J.O.J.; CHRISTOFARI, L.F. et al. Melhoria organizacional na produção de bezerros de corte a partir dos centros de custos. Rev. Bras. Zootec., v.37, p.580-587, 2008.

PACHECO, P.S.; RESTLE, J.; VAZ, F.N. et al. Avaliação econômica da terminação em confinamento de novilhos jovens e superjovens de diferentes grupos genéticos. Rev. Bras. Zootec., v.35, p.309-320, 2006.

PORTO, M.O.; PAULINO, M.F.; DETMANN, E. et al. Oferta de suplementos múltiplos para tourinho Nelore na fase de recria em pastagens durante o período da seca: desempenho produtivo e características nutricionais. Rev. Bras. Zootec., v.40, p.2548-2557, 2011.

REIS, R.A.; RUGGIERI, A.C.; CASAGRANDE, D.R. et al. Suplementação da dieta de bovinos de corte como estratégia do manejo das pastagens. Rev. Bras. Zootec., v.38, p.147-159, 2009.

STATISTICAL Analisys Sistem - SAS Institute Inc. SAS ${ }^{\circledR}$ User's Guide: Statistics, Version 9 Edition. Cary, NC: SAS Institute Inc., 2002. 